\title{
Biofabrication in Microfluidics: A Converging Fabrication Paradigm to Exploit Biology in Microsystems
}

\section{Xiaolong Luo*}

Institute for Bioscience and Biotechnology Research, University of Maryland, USA

\section{Editorial}

Biofabrication has emerged as a 21 st century manufacturing paradigm. The term of Biofabrication was first reported in 1994 by Fitz et al. [1] in biomineralization of pearls with biological mechanisms. In recent years, biofabrication has gained a broad recognition with the exponentially increased number of published papers and citations on this subject, as shown in Figure 1 and prompted the launch of the IOP journal Biofabrication in 2009. According to the journal, biofabrication uses cells or biologics as the basic building blocks to manufacture biological models, systems, devices and products [2]. A tutorial article by Mironov et al. [3] in 2009 defined biofabrication as the production of complex living and non-living biological components from raw materials such as living cells, molecules, extracellular matrices and biomaterials. An alternative definition was given by Payne and his coworkers in 2010, which defined biofabrication as the use of biological or biomimetic materials and mechanisms for construction [4]. Regardless of the slight emphasis of the definitions, there are several unique features of biofabrication: first, the building blocks are cells or biologics; second, the fabrication processes are bio-inspired or bio-friendly; and finally, the products are biological systems, models or devices with transformative properties.

Biofabrication is considered as a convergent fabrication
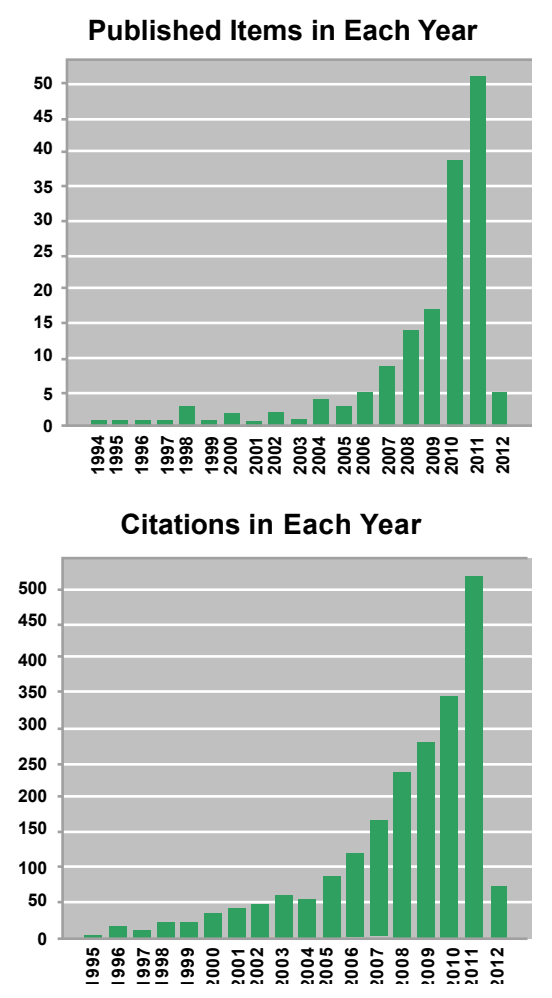

Figure 1: Publication and citation reports on the topic of biofabrication from ISI Web of Science. paradigm that bridges biotechnology and microfabrication. The last century has witnessed spectacular advances in both biotechnology and microfabrication of electronics. The potential of integrating microfabrication with biology is enormous. It may enable us to perform powerful biosensing and Lab-on-a-chip analysis in biomedical applications, to develop novel biofuel cells that can generate electricity from organic wastes and to deliver personalized medicines and restore functions with implantable devices in our bodies. One key challenge to integrate microfabrication with biology, however, is the divergent fabrication gap [4]. Fundamentally, transfer of signals in biology is via ions and small molecules, while in microfabricated devices the transmitting components are electrons and photons. Therefore, microfabrication uses top-down methods to pattern surfaces with permanent features. Biological components are labile and the construction starts with bottom-up genetic information to code amino acid sequence and protein folding. Microfabricated devices are created with error-free methods to fabricate defect-free devices, while biology has error-correction and healing mechanisms. As such, the use of biological components and mechanisms for construction represents an enabling strategy to interface microfabrication with biology.

One essential component to interface microfabricated devices with biology is stimuli-responsive biomaterials. The stimuli from the devices can be electrical [5], chemical [6], thermal [7] and photonic [8] signals including changes in $\mathrm{pH}$, ionic strength, temperature, light, oxidation/ reduction state, presence of small molecules, or the catalytic activity of enzymes. These device-imposed stimuli provide signals to initiate the device-biology integration and more importantly, to actively program the integration process with spatial-temporal controls. Stimuli-responsive biomaterials recognize and respond to these deviceimposed signals and facilitate the sequential assembly of biological components through direct molecular recognition (e.g., self assembly) and biochemical reactions (e.g., enzymatic assembly). The stimuliresponsive materials can be bio-friendly synthetic polymer systems [9], proteins such as collagen [7], polysaccharides such as chitosan [10] and alginate [11], peptides such as Fmoc [12], or materials constructed with peptides (responsive systems based on helical motifs, $\beta$-sheet structures, $\beta$-hairpin formation, amphiphilic monomers or amphiphilic

*Corresponding author: Xiaolong Luo, Institute for Bioscience and Biotechnology Research, University of Maryland, 2213 J.M. Patterson Building, College Park, MD 20742, USA, E-mail: xlluo@umd.edu

Received February 15, 2012; Accepted February 15, 2012; Published February 17, 2012

Citation: Luo X (2012) Biofabrication in Microfluidics: A Converging Fabrication Paradigm to Exploit Biology in Microsystems. J Bioengineer \& Biomedical Sci 2:e104. doi:10.4172/2155-9538.1000e104

Copyright: @ 2012 Luo X. This is an open-access article distributed under the terms of the Creative Commons Attribution License, which permits unrestricted use, distribution, and reproduction in any medium, provided the original author and source are credited. 
monomers bearing aromatic groups [13]). As such, biofabrication techniques encompass a broad range of physical, chemical, biological and/or engineering process.

Biofabrication in microfluidics is a perfect representation of integrating microfabricated devices with biology. Microfluidics deals with behavior, control and manipulation of fluids on the micrometer and sub-micrometer scale that integrates one or multiple functions into a single microfabricated chip. By consuming less fluidic volume and offering faster and parallel process control, advances in microfluidic technology are revolutionizing integrated platforms with applications in biology, biochemistry, medicine and biotechnology studies, among many others. Biofabrication in microfluidics encompasses the benefits of microfluidic technology to construct biological systems that offers unprecedented capabilities. Highlighted here are some recent examples of biofabrication in microfluidics with device-imposed (1) electrical signals, (2) photonic signals and (3) flow-driven chemical gradients to guide the assembly of polysaccharides including chitosan, alginate and collagen.

\section{Biofabrication with Electrical Signals in Microfluidics}

The first example of biofabrication in microfluidics uses electrical signal to guide the assembly of chitosan for subsequent construction of metabolic pathway enzymes to reconstruct signaling molecule synthesis pathway on a chip [14]. Chitosan is a linear polysaccharide derived from the naturally second abundant biopolymer chitin. One unique property of chitosan is that it has an amine group on each deacetylated sugar ring. The amine groups are protonated in acidic solution. But when $\mathrm{pH}$ is above the pKa value of 6.3, chitosan molecules are deprotonated and become insoluble. When negative electric signal is applied to the electrode in chitosan solution, proton consumption occurs at the cathode and proton consumption is compensated with water hydrolysis. As the hydroxyl ions accumulate, a $\mathrm{pH}$ gradient is generated near the surface of negative electrode. Chitosan molecules are then deprotonated and immobilized on the electrode surface [15]. By genetically engineering a pentratyrosine tag to model proteins and signal synthesis enzymes or complex structures containing multiple enzymes, enzymatic pathways were reconstructed in microfluidics. Signalling molecules was locally synthesized and induced populationscale cellular response of spatially assembled bacteria. As such, biofabrication with chitosan enables the hierarchical assembly of biological components onto microfabricated devices [16], while the biological components perform biological functions in a controlled sometimes enhanced manner with the advantages of microfluidic systems [14].

In the second example, the electrodeposition of $\mathrm{pH}$-responsive chitosan was extended to exploit the electrodeposition of alginate, an anionic polysaccharide derived from the cell walls of brown algae and has been widely used for cell immobilization in tissue engineering. One unique property of alginate is that the homopolymeric blocks of guluronate residues can be crosslinked by divalent cations such as calcium ions into hydrogel network. Before the electrodeposition process, soluble sodium alginate is first blended with insoluble calcium carbonate particles (in nanometer scale). When anodic electrical signal is applied to the electrode in the alginate calcium carbonate suspension, protons are generated with water hydrolysis at the electrode surface and form local low $\mathrm{pH}$ region. Calcium ions are then locally released and crosslink alginate molecules into hydrogel. One important application of electrodepositing alginate hydrogel in microfluidics is to assemble cell populations with spatial programmability. When target cells are blended into the alginate solution, the applied electrical signal guide the entrapment of target cells within the hydrogel with fine spatial resolution. Assembly of different cell populations can be achieved by sequentially introducing distinct cell solutions into the microfluidic channels and applying electrical signals to the same or different electrodes [17].

In a final example of biofabrication in microfluidics with electrical signals, electrochemically inactive collagen was exploited to grow suspended membranes in capillaries between two electrodes [18]. Collagen is the most abundant mammalian protein, with $\mathrm{pH}$ dependent surface charges. The amino acids are protonated in acidic condition and deprotonated in alkaline condition. Therefore, by applying substantial high potential to induce $\mathrm{pH}$ gradients near the electrode surfaces, collagen molecules migrate away from the two facing electrodes and aggregate as hydrogel, or suspended membranes in the middle of the electrolyte bath or capillary tubes. Different from the case of chitosan electrodeposition where the solidification takes place at the cathode surface, the electrochemically driven migration is two-directional away from both anode and cathode surfaces towards the middle.

\section{Biofabrication with Photonic Signals in Microfluidics}

Biofabrication in microfluidics can also take place with light signals, which can be easily patterned through a photo-mask. In the first example, alginate gel was formed in select regions of a microfluidic device through light-triggered release of caged calcium. Calcium is initially chelated by DM-nitrophen (DM-n) in the pre-gelled alginate solution. After sufficient UV exposure the caged calcium is released from DM-n causing alginate to cross-link alginate into hydrogel. To reverse the process, the cross-linked alginate can easily be dissolved by EDTA [19]. A simpler version of implementing light signal without using the expensive caged calcium is to exploit the approach of introducing an insoluble salt of the cation (e.g., calcium carbonate) into an aqueous solution of the polymer (e.g., alginate). Furthermore, a photo acid generator (PAG) was also mixed into the suspension. Upon UV irradiation, the PAG dissociates to release hydrogen ions, which react with the calcium carbonate to generate free $\mathrm{Ca}^{2+}$. In turn, the calcium ions cross-link the alginate chains into a physical network, thereby resulting in a hydrogel [20]. In an alternative system, electrodeposition of calcium alginate hydrogels is performed by using a digital micromirror device (DMD) with the same calcium carbonatesuspended alginate solution [21]. A photoconductive substrate serves as a photo-anode to produce protons, which can lead to a decreased $\mathrm{pH}$ gradient. The low $\mathrm{pH}$ generated at the anode then locally releases calcium ions from insoluble calcium carbonate and crosslink alginate into hydrogels. One advantage of light signal over electrical signal is the flexibility of changing a design without the need of fabricating new devices. On the other hand, the control of light through devices is not as straightforward as the control of electrical signals.

\section{Biofabrication with Flow-Driven Chemical Gradients in Microfluidics}

Biofabrication with electrical and photonic signals is convenient, fast and can be programmed by controlling the device-imposed or external stimuli. Meanwhile, there are many cases that the electrical signal or UV light and the created $\mathrm{pH}$ gradients are not perfectly cellfriendly. Recently, flow-driven chemical gradients in microfluidics have also been exploited to biofabricate alginate hydrogels [22] and freestanding chitosan membranes [23]. Given the nature of laminar flow inside microfluidic channels, in situ generation of $\mathrm{pH}$ and 
chemical (e.g., $\mathrm{Ca}^{2+}$ ) can be easily generated at the follow interfaces in a microfluidic network. In the first example, the alginate hydrogel is created by combining two laminar flows of the gel precursor solutions-a calcium solution and an alginate solution-in a microchannel. A separation flow between the alginate and calcium solutions is used to handle the flow protocol: Calcium ions are initially separated by the separate flow from the alginate flow streams. Once the separation flow is stopped, a gradient of calcium ion diffuses into the alginate solutions and crosslink alginate into hydrogel, which allows a gel bar composed of many layers, each layer filled with a certain particle type, to be formed [22]. In the second example, a $\mathrm{pH}$ gradient is in situ generated at the flow interface between an acidic chitosan solution and a basic buffer solution by balancing the pressure between the two flow streams. Chitosan molecules are then deprotonated and solidify at the flow interface to form a semi-permeable, freestanding chitosan membrane [23]. Additionally, stratified alginate hydrogels with distinct target cell populations can be assembled along the pre-fabricated chitosan membrane to form distinct cell-gel layers [24]. With the fine control of spatial resolution, semi-permeability to small molecules and convenient fluidic and optical access to both sides of the freestanding membranes and hydrogels, the stratified cells layers assembled in microfluidic system offers ideal platform for cell-cell interaction and signaling studies. Importantly, cells assembled with the flow-driven chemical gradients are not exposed to either electrical signal or UV light. Therefore, this biofabrication strategy is particularly suitable for applications where cells are sensitive to $\mathrm{pH}$ change or UV light.

In summary, biofabrication in microfluidics offers a converging fabrication paradigm to exploit biology in microsystems. Stimuliresponsive biomaterials are essential for signal-guided hierarchical assembly of biological components onto microfabricated devices. Transformative new platforms created with biofabrication in microfluidics offer unprecedented capabilities to investigate biology with precise control and active programmability. It is envisioned that biofabrication in microfluidics will be implemented to a wide spectrum applications ranging from replication of metabolic pathways as testbeds for drug discovery to direct observation and active manipulation of cell-cell interaction studies and to construction of tissues- and organson-a-chip replications.

\section{References}

1. Fritz M, Belcher AM, Radmacher M, Walters DA, Hansma PK, et al. (1994) Flat pearls from biofabrication of organized composites on inorganic substrates. Nature 371: 49-51.

2. Sun W (2009) Welcome to Biofabrication. Biofabrication 1: 010201.

3. Mironov V, Trusk T, Kasyanov V, Little S, Swaja R, et al. (2009) Biofabrication: a 21st century manufacturing paradigm. Biofabrication 1: 022001.

4. Liu Y, Kim E, Ghodssi R, Rubloff GW, Culver JN, et al. (2010) Biofabrication to build the biology-device interface. Biofabrication 2: 022002.

5. Wu L-Q, Gadre AP, Yi H, Kastantin MJ, Rubloff GW, et al. (2002) VoltageDependent Assembly of the Polysaccharide Chitosan onto an Electrode Surface. Langmuir 18: 8620-8625.

6. Workman VL, Dunnett SB, Kille P (2008) Palmer DD. On-chip alginate microencapsulation of functional cells. Macromol Rapid Commun 29: 165-170.

7. Cross VL, Zheng Y, Won Choi N, Verbridge SS, Sutermaster BA, et al (2010) Dense type I collagen matrices that support cellular remodeling and microfabrication for studies of tumor angiogenesis and vasculogenesis in vitro. Biomaterials 31: 8596-8607.

8. Pregibon DC, Toner M, Doyle PS (2007) Multifunctional encoded particles for high-throughput biomolecule analysis. Science 315: 1393-1396.

9. Stuart MA, Huck WT, Genzer J, Müller M, Ober C, et al. (2010) Emerging applications of stimuli-responsive polymer materials. Nat Mater 9: 101-113.
10. Yi H, Wu LQ, Bentley WE, Ghodssi R, Rubloff GW, et al. (2005) Biofabrication with chitosan. Biomacromolecules 6: 2881-2894.

11. Shi XW, Tsao CY, Yang XH, Liu Y, Dykstra P, et al. (2009) Electroaddressing of Cell Populations by Co-Deposition with Calcium Alginate Hydrogels. Advanced Functional Materials 19: 2074-2080.

12. Liu Y, Cheng Y, Wu HC, Kim E, Ulijn RV, et al. (2011) Electroaddressing agarose using Fmoc-phenylalanine as a temporary scaffold. Langmuir 27 7380-7384.

13. Mart RJ, Osborne RD, Stevens MM, Ulijn RV (2006) Peptide-based stimuliresponsive biomaterials. Soft Matter 2: 822-35.

14. Luo X, Lewandowski AT, Yi H, Payne GF, Ghodssi R, et al. (2008) Programmable assembly of a metabolic pathway enzyme in a pre-packaged reusable bioMEMS device. Lab Chip 8: 420-430.

15. Cheng Y, Luo XL, Betz J, Buckhout-White S, Bekdash O, et al. (2010) In situ quantitative visualization and characterization of chitosan electrodeposition with paired sidewall electrodes. Soft Matter 6: 3177-3183.

16. Yi H, Wu LQ, Bentley WE, Ghodssi R, Rubloff GW, et al. (2005) Biofabrication with chitosan. Biomacromolecules 6: 2881-2894.

17. Cheng Y, Luo X, Tsao CY, Wu HC, Betz J, et al. (2011) Biocompatible multiaddress $3 \mathrm{D}$ cell assembly in microfluidic devices using spatially programmable gel formation. Lab Chip 11: 2316-2318.

18. Baker HR, S EF, Poduska KM (2008) Electrochemically controlled growth and positioning of suspended collagen membranes. Langmuir 24: 2970-2972.

19. Chueh BH, Zheng Y, Torisawa YS, Hsiao AY, Ge C, et al. (2010) Patterning alginate hydrogels using light-directed release of caged calcium in a microfluidic device. Biomed Microdevices 12: 145-151.

20. Javvaji V, Baradwaj AG, Payne GF, Raghavan SR (2011) Light-activated ionic gelation of common biopolymers. Langmuir 27: 12591-12596.

21. Huang SH, Hsueh HJ, Jiang YL (2011) Light-addressable electrodeposition of cell-encapsulated alginate hydrogels for a cellular microarray using a digital micromirror device. Biomicrofluidics 5 .

22. Johann RM, Renaud P (2007) Microfluidic patterning of alginate hydrogels Biointerphases 2: 73-79.

23. Luo X, Berlin DL, Betz J, Payne GF, Bentley WE, et al. (2010) In situ generation of $\mathrm{pH}$ gradients in microfluidic devices for biofabrication of freestanding, semipermeable chitosan membranes. Lab Chip 10: 59-65.

24. Luo X, Wu H-C, Tsao C-Y, Cheng Y, Betz J, et al. Biofabrication of stratified biofilm mimics for observation and control of bacterial signaling. Biomaterials. 